Fecha de recepción: noviembre 2020 Fecha de aprobación: diciembre 2020 Fecha publicación: marzo 2021

\section{La trama en el espacio tridimensional. La aplicación de un recurso gráfico complementario en el nuevo edificio de la Fondation Louis Vuitton}

María Laura Spina ${ }^{(1)}$

Resumen: En el diseño de un Sistema de Identidad Visual de Alta Complejidad, el uso y aplicación de un repertorio de identificadores secundarios otorga valor a la identidad misma.

La aplicación de tramas bidimensionales aplicadas a espacios tridimensionales impacta eficazmente en la identidad de la empresa, resignificando el propio Sistema Visual convirtiendo al espacio arquitectónico en un hito señalético corporativo dentro del Programa Tridimensional.

Actualmente, marcas de Indumentaria reconocidas, recurren al dominio del espacio bitridimensional con el objetivo de convertirse en símbolos gráficos universales de alto poder de recordación dentro de un espacio urbano.

Palabras clave: arquigrafía - bidimensión - estructura - geometría - hito - identidad - módulo - señalética identitaria - trama - tridimensión.

[Resúmenes en inglés y portugués en la página 142]

(1) Diseñadora Gráfica Universidad de Buenos Aires. Profesora de la Universidad de Palermo en el Departamento de Diseño Visual y Comunicación Corporativa y Empresaria de la Facultad de Diseño y Comunicación.

\title{
Introducción
}

La Fondation Louis Vuitton es un museo construido por el reconocido arquitecto Frank Gehry con el objetivo de promover el arte y la cultura. Fue inaugurado en octubre de 2014 y se encuentra enclavado en el entorno de Bois de Boulogne de París.

El diseño evoca a aquellos edificios de vidrio de los jardines del siglo XIX. Se encuentra construido junto a un jardín de agua y consta de un conjunto de bloques blancos denominados icebergs rodeados por doce velas de vidrio con el apoyo de vigas de madera. 
La forma del edificio se caracteriza por sus formas cóncavas y convexas y los materiales utilizados (acero, hormigón, madera y vidrio) han sido sometidos a la contorsión geométrica, plegándose y curvándose, gestando una trama visual tridimensional de gran riqueza expresiva. Los módulos rectangulares de esta trama se disponen de manera regular en el espacio tridimensional conformando tramas regulares y unitarias pues poseen un único motivo como figura que produce la partición infinita del espacio.

El edificio posee una arquitectura vidriada con forma de velero. Las velas que lo componen le dan a la Fondation Louis Vuitton transparencia y sensación de movimiento, permitiendo que el edificio refleje el agua y los jardines y que la luz cambie permanentemente. Los volúmenes que lo conforman están separados por aberturas, huecos y superposiciones rodeados por paredes de cristal. Cada una de estas velas, con forma y curvatura diferentes, se sostienen por vigas de acero y madera y están compuestas por paneles de vidrio serigrafiado que permiten reflejar la energía luminosa que reciben ofreciendo hermosas vistas del paisaje integrándolo como experiencia de museo.

El museo es un lugar de encuentro, donde se realizan exposiciones, seminarios, debates y espectáculos y porta la marca insignia Louis Vuitton resignificando la identidad de la misma.

\section{Arquitectura corporativa y señalética}

El proyecto señalético es único y específico en cada caso y está al servicio de quienes quieran utilizarlo. La arquitectura de la Fondation Louis Vuitton es parte de la señalética y subyace en ella la identidad corporativa, la imagen de marca.

La señalética responde a la necesidad de información y orientación, atravesada por el fenómeno de la movilidad social y la proliferación y diversificación de servicios.

Esta dinámica social recoge la idea de circunstancialidad, es decir el paso de los individuos por determinados espacios con una permanencia esporádica, aleatoria, efímera, considerada una actividad de naturaleza itinerante.

El entorno diseñado y construido es considerado un enorme contenedor de vida. Allí, la gente, convive con los objetos, arte, diseño y tecnología.

La función de la señalética orientativa implica la presencia del usuario sumado al diseñador o arquitecto (quien orienta) y la empresa, la mencionada Louis Vuitton, quien es quien quiere orientar.

Teniendo en cuenta estos aspectos, puntualizar este espacio singular en la ciudad de París es una estrategia comunicacional de gran visibilidad en la vía pública. Señalar la identidad del lugar, la empresa, y coordinar la orientación interna para el visitante, el usuario y la prestación del servicio es la función de la señalética corporativa.

La presencia de arquitecturas singulares en las ciudades puede observarse en todo el mundo. Las empresas y grandes corporaciones recurren a ellas con el objetivo de afirmar la vida urbana. También quienes producen ciudades promueven esta arquitectura institucional poco convencional como estrategia de atracción turística, financiera y cultural.

El museo de la Fondation Louis Vuitton participa del diseño urbanístico y arquitectónico aportando valor a la imagen de la ciudad. 


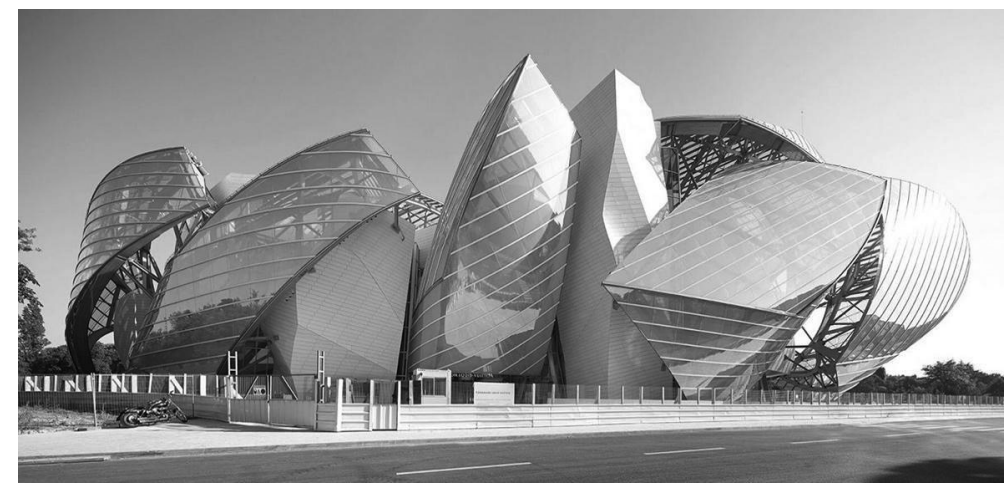

Figura 1. Museo de la Fondation Louis Vuitton. Fuente: http:/plataformaarquitectura.cl/

Considerado un edificio singular, insólito y emblemático, creado por un arquitecto innovador, Frank Gehry, este museo se encuentra a la altura del Centro Pompidou en París o del Museo Guggenheim de Bilbao, entre otros. El auge económico, cultural y tecnológico de estas obras dejan su impronta en la imagen del país, contribuyendo a la señalética corporativa. De este modo, la identidad de la empresa Louis Vuitton forma parte de la ciudad de París reforzando su imagen frente al mundo (Figura 1).

\section{Señalética identitaria:}

Con la aparición en los países más desarrollados del turismo cultural, los museos, las exposiciones internacionales y las nuevas experiencias turísticas, se hace cada vez más visible la atención de la necesidad de información - orientación - motivación impulsada por la exigencia de atender al público en lugares de servicios, organizando los espacios para que éstos sean accesibles y utilizables.

El museo de la Fondation Louis Vuitton ha sido concebido bajo estos aspectos ya que permiten orientar los flujos en sus propios espacios para una mejor información y eficacia, aportándole a la empresa rasgos exclusivos y diferenciadores: sus rasgos de identidad.

Esta señalética identitaria funciona in situ, en el mismo entorno urbano, permitiendo la identificación y reconocimiento del público a través de una fuerte presencia física, formando parte de la vida cotidiana de la gente.

Esta obra arquitectónica inserta en el entorno, irradia su presencia, posee una forma identificatoria distinta, novedosa, competitiva. Su propia cáscara o contorno (volúmenes contenedores de una trama visual tridimensional) la encierra y su lugar interior se vuelve acotado y limitado. Su dintorno, es el espacio donde se aglutina y se presta servicio al público, donde éste experimenta la satisfacción del servicio y las nuevas experiencias emocionales. Este edificio contribuye a destacar o potenciar la imagen pública o imagen de marca que 
se quiere transmitir. Este marcaje sobrepasa el espacio físico frecuentado por los diferentes públicos y se extiende a múltiples soportes de manera coherente.

La presencia de la identidad en la vía pública, en un contexto urbano, en una sociedad, permite observar la presencia constante y permanente de la identidad corporativa en la vía pública. La calidad de la imagen corporativa con el excelente diseño arquitectónico del museo y las nuevas tecnologías, la señalización física de este espacio como distintivo de una identidad exclusiva, su competitividad que está dada por su ubicación en un emplazamiento estratégico, la visualización absoluta asociando servicio al negocio y, finalmente, el control directo de la empresa sobre el conjunto de elementos del inmueble como estado material, iluminación y posibilidad de intervenciones puntuales se asocian para reforzar la identidad de este espacio. Tal como explica Joan Costa:

La idea de integrar todas las comunicaciones de la empresa y la necesidad de éstas de hacerse más presentes en su inserción a la sociedad a la cual sirven, junto con la revalorización de antiguos modos de comunicar la Identidad, ha llevado a las empresas a recuperar la función de "señalación" de sus inmuebles -sede central, puntos de venta, franquicias, delegaciones, sucursales, etc.- unificando sus sistemas de identidad visual corporativa. Esta disciplina estratégica conseguirá para la empresa una sola imagen y una sola Voz, y hará coherentes todas las manifestaciones y posesiones de la empresa, desde la arquitectura y los productos/servicios a la información. La empresa recupera así su faceta más genuinamente institucional o corporativa (Costa, 2007, p. 125).

El entorno diseñado (paisajismo, urbanismo y escenarismo) y construido (la arquitectura y el interiorismo) es considerado un contenedor de vida, donde la gente convive con el diseño de manera directa, con los objetos diseñados, los mensajes y las tecnologías.

En este entorno del museo de la Fondation Louis Vuitton, el edificio en sí mismo cumple una función orientativa ya que participa en el juego de dos extremos: quien orienta y quien es orientado.

El usuario, visitante o público es activo e imprescindible. Se le brindan motivaciones por parte de quien orienta, en este caso el arquitecto Frank Gehry y su equipo, y de quien quiere orientar, la Maison Louis Vuitton.

Se observa el cumplimiento de diferentes variantes: la orientación al ciudadano pues el edificio mismo, por su envergadura, funciona como faro en la ciudad luz. Quien se acerque como peatón o motorizado será testigo de esta arquitectura señalética urbana. Además el usuario será orientado hacia un servicio señalado como lugar singular, visible y de gran competencia en la vía pública siendo reforzada la identidad de este espacio de museo a través de su imagen y sus acciones hacia el visitante.

Dentro del ámbito interno del museo se percibe un lugar de encuentro, relaciones y vínculo, donde se coordina la orientación interna para el visitante y el usuario a quien se le presta el servicio. Existe, con mucha precisión, una continuidad de excelencia entre exterior e interior, información y servicios de calidad, asociación entre calidad del servicio, ambiente y empresa y entorno envolvente en el museo identificado como un lugar de notable experiencia emocional. 


\section{La adaptación de la señalética al medio:}

Todo espacio de acción obedece a una función precisa ya que está definido por sus funciones sociales. Existen determinados códigos que forman parte de un nivel de conocimiento constituido por fragmentos de cultura y que implican, en sí mismos, una convención social. Así como un hospital es pensado por los individuos como silencioso, tranquilo, luminoso, funcional, higiénico y limpio, el museo de la Fondation Louis Vuitton es pensado espacioso, multicultural, luminoso, innovador y creativo.

No debe dejarse de lado la estructura arquitectónica. Un hospital, puede ser simple y transparente o muy complejo, opaco, laberíntico a los ojos del usuario. De acuerdo a la medida de esta simplicidad o complejidad, la señalética se ve afectada y debe dar respuesta correcta al espacio donde se va a insertar.

También el estilo ambiental avanza yendo desde lo general a lo particular. Así como un hospital puede ser grande, transparente y luminoso, también puede percibirse frío y distante. El museo de la Fondation Louis Vuitton se muestra grande, luminoso y diferente. Es percibido como cálido y funcional de acuerdo a su arquitectura exterior y su ambientación interior.

Recopilando los aspectos más interesantes se observan: el espacio en general del museo entendido como espacio total (edificio más entorno) y espacio parcial (conjunto de bloques vidriados del museo), la morfología arquitectónica y de entorno referida a la forma misma del edificio y al tratamiento gráfico y volumétrico del mismo.

El edificio está construido en dos plantas, contiene once galerías de diferente tamaño para exponer arte contemporáneo, colecciones, exposiciones e intervenciones de artistas, un auditorio con configuraciones modulables en planta baja y terrazas en los techos a varios niveles para instalaciones de arte y diversos eventos

Otro aspecto es la organización del espacio en función de los servicios que se prestan al público, ya que su función no es solamente dedicarse al arte contemporáneo sino que el espacio es elegido para realizar debates, seminarios, clases magistrales, coloquios y espectáculos en vivo, cine y video. También la fundación realiza pedidos especiales a artistas.

No deben dejar de tenerse en cuenta las distancias de visión del visitante hacia el edificio y hacia los paneles interiores según su tamaño y contraste, ni la iluminación ambiente con la luz natural que atraviesa los paneles de cristal de las velas de un velero imaginario que parecen infladas por el viento, ni la imagen de marca del espacio objeto de tratamiento señalético que se ve reforzada por un gran logotipo de acero inoxidable con las iniciales de la empresa LV, diseñado por Frank Gehry.

\section{El mundo tridimensional:}

El mundo en el que el ser humano habita posee tres dimensiones, largo, ancho y una profundidad física denominada tercera dimensión.

Para comprender este mundo tridimensional, el objeto, debe poder ser visto desde diversos ángulos y distancias diferentes, para luego, reunir en la mente toda la información posible para comprender dicha realidad tridimensional. 


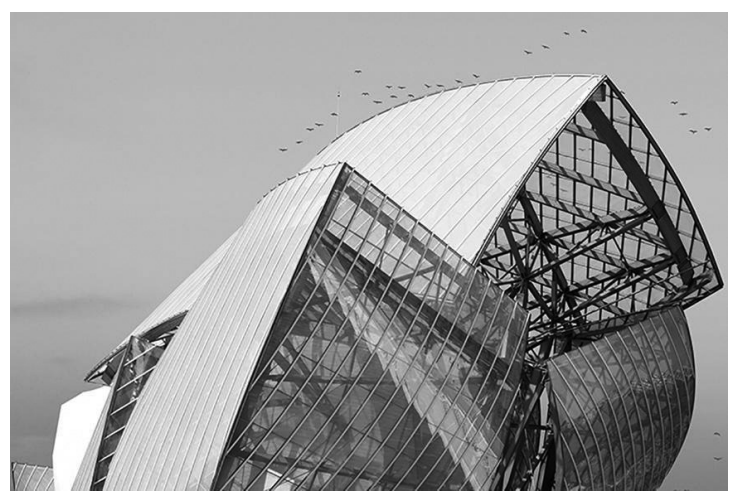

Figura 2: volumen translúcido. Fuente: http:/fondationlouis vuitton.fr/

Es a través de la mente humana que el museo de la Fondation Louis Vuitton con sus volúmenes translúcidos y la trama aplicada en ellos puede ser comprendido en su plenitud obteniendo su significado más profundo. Esta tridimensionalidad procura generar armonía y orden visuales considerando las diversas perspectivas desde ángulos distintos a través de la reunión de formas y materiales tangibles enmarcados en un espacio real.

La experiencia de transitar este espacio permite entender al museo como una obra escultórica con estructuras internas y externas que van más allá de la comprensión atrayendo al ojo del espectador a través de la trama aplicada y el color dotando a la superficie de una textura donde el volumen y el espacio son muy valiosos (Figura 2).

\section{Elementos constructivos del diseño tridimensional:}

Estos elementos son importantes ya que poseen fuertes cualidades de estructura. Se componen de vértices, filos y caras. Es en los vértices donde convergen diversos planos en un punto conceptual; cuando dos planos paralelos se unen a lo largo de una línea conceptual, se producen los filos y cuando un plano se convierte en superficie da origen a las caras, las cuales encierran un volumen.

Estos elementos constructivos ayudan a definir las formas volumétricas permitiendo visualizar una forma o apariencia visual total de un diseño.

El museo Vuitton posee formas volumétricas con aplicación de trama, con una estructura simple que gobierna la forma construida y actúa como esqueleto que da sostén a todo el volumen dando origen a una apariencia externa compleja de gran riqueza visual.

Cada módulo de la trama se convierte en una célula espacial en la estructura de la pared. Estas células están dispuestas de manera bidimensional sobre los planos frontales de los volúmenes que conforman el museo. Dichas estructuras bidimensionales actúan como estructuras de pared ya que poseen cierta profundidad dando origen a las tramas tridimensionales. El museo posee células espaciales cuyos planos exteriores han sido construidos 
para que no estén en ángulos rectos entre sí pues siguen el movimiento o la curvatura de los volúmenes que componen toda la estructura.

Es importante destacar el tratamiento de los filos ya que la desviación de los filos paralelos cambia la rectangularidad de las figuras en las caras de los volúmenes del museo. En ocasiones esto conduce a la deformación de las caras gestando rasgos interesantes.

\section{La estructura lineal:}

El museo de la Fondation Louis Vuitton con su conjunto de bloques blancos conocidos como icebergs revestidos con paneles de hormigón reforzado con fibras y rodeados por velas de vidrio translúcidas envueltas en una trama visual con apoyo de vigas de madera aporta transparencia y sensación de movimiento.

Para la construcción de estas formas geométricas sólidas, de caras planas y filos rectos, es necesario cortar las figuras de las caras (en este caso son los rectángulos que componen la trama de manera modular), pegarlas entre sí dando origen a una estructura lineal y cuidando las uniones, las cuales deben ser fuertes y resistentes para dar sostén a toda la estructura. Aplicando la repetición de cada módulo, unidad o célula espacial hacia arriba y abajo, manteniendo la figura, el tamaño y la dirección de cada módulo se obtiene una estructura vertical y horizontal de filos rectos ya que se fueron colocando los módulos uno sobre otro o uno al lado del otro.

\section{La trama bi-tridimensional:}

La trama es la resultante de la partición del plano, donde, al repetirse el motivo se provoca un ritmo visual.

Desde otro punto de vista, la trama es considerada una repetición de módulos, los cuales, al repetirse, generan ritmo y armonía visual. La combinación de la forma del módulo, tamaño, color, textura, dirección y articulación entre los mismos módulos provocan diferentes efectos visuales.

La trama conformada por estos módulos de repetición, origina una metodología secuencial dando vida a una serie o conjunto de elementos relacionados entre sí, sucediéndose unos a otros según un criterio o ley predeterminada. Con respecto a la estructura compositiva modular, Gonzaléz Ruiz escribió:

Los módulos pueden repetirse y disponerse según determinadas leyes organizativas de las partes, al servicio de la construcción de un todo formal. Llamaremos estructura compositiva entonces, a la disciplina ordenadora que subyace en la serie modular (González Ruiz, 1994, p. 273).

Es en la observación de la naturaleza donde se observa orden y armonía. Lo simétrico, equilibrado o bien proporcionado, donde el orden regular entre las formas pone en evidencia operaciones visuales y sus múltiples combinaciones permiten abordar la lectura del 


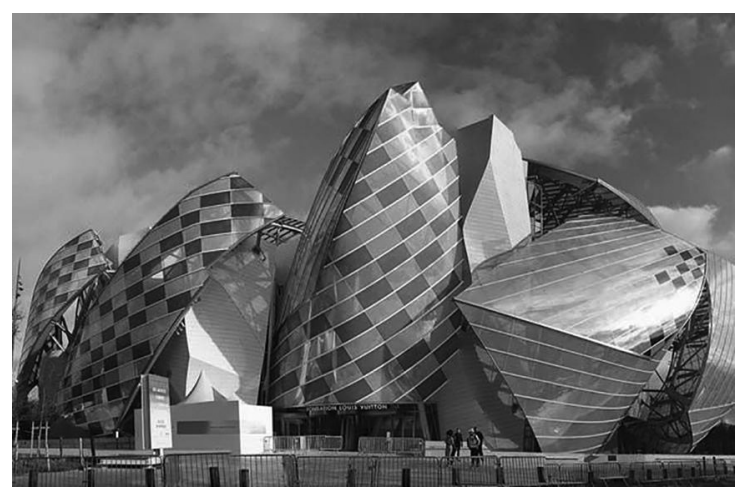

Figura 3. Trama

aplicada en arquitectura exterior. Fuente: http:/ fondationlouisvuitton.fr/

museo de la Fondation Louis Vuitton desde otra perspectiva. En su arquitectura espacial, las conformaciones o particiones del espacio denominados motivos o módulos dan lugar a tramas espaciales que son percibidas por el público como una tridimensionalidad ya que están aplicadas a los volúmenes de vidrio que componen la arquitectura de este edificio (Figura 3).

La topología, ciencia que constituye una nueva parte de la geometría y que permite analizar las formas y la posición respectiva de las figuras geométricas en función de criterios mucho más generales que en la geometría elemental, permite redefinir el espacio del museo de la Fondation Luis Vuitton como un lugar donde la posición y la disposición de las figuras geométricas en la superficie bidimensional se adapta de manera natural al espacio tridimensional. De este modo, se observa la creación de series modulares que responden a un criterio estructural de ordenamiento de aquellos módulos similares o iguales entre sí, los cuales mantienen el orden y la armonía visual. La trama, en este espacio, surge de la repetición de una forma unitaria o módulo de acuerdo a un determinado principio serial dando lugar a la seriación modular.

\section{Las técnicas visuales en la composición de la trama:}

Las técnicas visuales permiten ordenar con claridad los elementos que se disponen en el espacio con el objetivo de fortalecer el mensaje que se quiere dar. Estas técnicas se potencian unas a otras al estar combinadas. Su aplicación y utilización debe ser clara y didáctica para evitar generar confusiones en el mensaje.

Dentro de las técnicas visuales, se destaca el equilibrio. Su importancia se basa en el funcionamiento de la percepción humana y en la necesidad de equilibrio que se mantiene en el diseño, en este caso, las velas tramadas del museo, como en la reacción del público ante este objeto arquitectónico de características tan particulares. Este equilibrio puede lograrse de dos modos, simétrica o asimétricamente. 


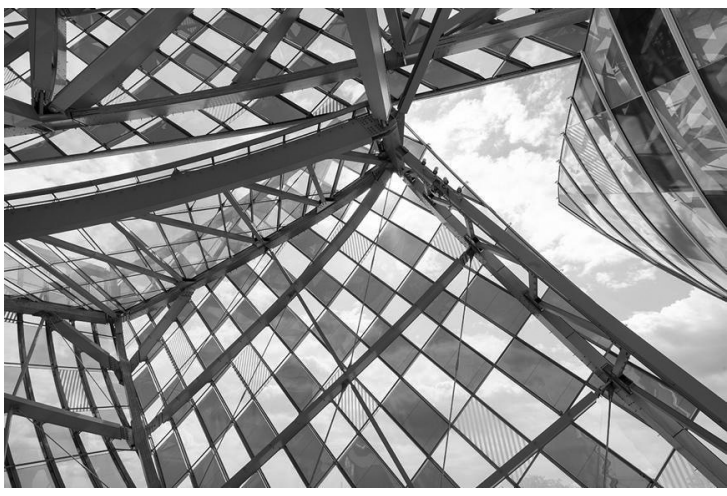

Figura 4. Módulo rectangular de la trama. Fuente:http:/ fondationlouisvuitton. $\mathrm{fr} /$

El motivo que se puede observar en el museo de la Fondation Louis Vuitton constituye una figura rectangular. Es la parte elemental de la organización simétrica y su aplicación se rige por las leyes u operaciones de simetría. Las mismas se refieren a la identidad o representación del objeto sobre sí mismo sin ninguna variación, la reflexión especular o retrato bilateral donde se invierten los lados, la traslación o corrimiento del motivo sobre un órgano recto denominado eje de traslación, la rotación o giro del motivo alrededor de un eje de rotación y la extensión o variación multiplicada del motivo pero que permanece semejante a sí mismo (Figura 4).

El museo posee rectángulos como motivos, operados por las leyes de identidad y traslación que se encuentran regulados según su equivalencia o la semejanza entre sus partes. Los módulos de esta trama operan en tres dimensiones: largo, ancho y profundidad. Estas tres direcciones primarias siguen una dirección vertical cuya lectura se desarrolla de arriba hacia abajo, una dirección horizontal que va de izquierda a derecha y una dirección transversal que va de adelante hacia atrás.

La regularidad de estos módulos favorecen la uniformidad de estos elementos y el desarrollo de un orden. La continuidad espacial refuerza la individualidad de cada una de las partes que componen la trama, siendo la secuencialidad la respuesta compositiva a un plan de representación que está dispuesto con un orden lógico. No se observa complejidad visual ni profusión de elementos aún en la presencia de numerosas unidades o motivos. Si bien, los rectángulos de la trama se aprecian con claridad, la imagen que proyectan es simple, no recargada y enriquecedora.

Otra de las técnicas visuales observadas es la unidad de los motivos rectangulares, aportando un equilibrio adecuado de dichos elementos en una totalidad que es muy perceptible de manera visual. También la transparencia implica un detalle visual a través del cual se puede visualizar el entorno, lo que está detrás es percibido por el ojo. Las velas del museo son transparentes y permiten visualizar y apreciar el espacio exterior a través de las mismas. Son sutiles, delicadas y refinadas, siendo la trama visual el envolvente de las mismas, actuando como una tela alrededor de un cuerpo. 


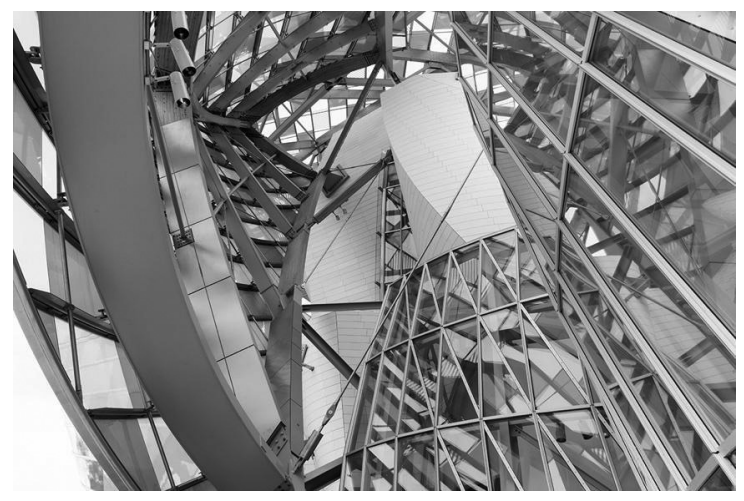

Figura 5. Trama

visual desde el

interior. Fuente: http:/

fondationlouisvuitton.fr/

La exageración en tamaño y forma se percibe en la morfología propiamente dicha de las velas arquitectónicas que componen el museo. Es una exageración visualmente efectiva, extravagante y atractiva ya que las mismas se pueden visualizar desde lejos intensificando las sensaciones y emociones del visitante. La profundidad en estos volúmenes, regida por el uso de la perspectiva se ve reforzada por las luces y las sombras sugiriendo una apariencia natural al museo en el emplazamiento donde se encuentra ubicado (Figura 5).

\section{La forma y el campo:}

La forma, enmarcada en el campo de la morfología del diseño gráfico puede ser estudiada como elemento y como organización. En ambos casos, pueden reconocerse diferentes modos de generación y organización visual, los cuales permiten arribar a la producción de diversos grados de complejidad.

Dentro del campo de la forma como elemento surgen las formas de generación aleatoria, producto de la práctica espontánea: grafismos, manchas, elementos naturales y aquellas formas surgidas del registro fotográfico con mayor o menor grado de iconicidad.

Desde otro punto de vista, en el campo de la geometría, surgen aquellas formas geométricas que dan origen a la mayor cantidad de formas utilizadas en diseño a través de diferentes operaciones como el desplazamiento, traslación o movimiento operado por una unidad geométrica. De este modo, si un punto se traslada da origen a una recta, si una recta se traslada da origen a un plano y si el plano se traslada da origen a un volumen.

Estas formas de generación geométrica poseen una estructura general articulada por tres estructuras básicas: la estructura portadora, modular y de proyección. Al operarlas, de manera combinada o separadas es posible obtener nuevas formas similares a las formas básicas pero ampliando el repertorio de formas posibles y ubicadas dentro de un mismo sistema formal. 
La estructura portadora se sostiene en el soporte portador de la forma y está constituida por los puntos y líneas que la conforman. En el caso de los módulos rectangulares que conforman la trama del edificio del museo, los mismos están articulados por sus lados, diagonales y ejes.

La estructura modular se forma por submúltiplos de la figura constituyendo un reticulado orientado según los lados del perímetro. En el caso de los rectángulos de la trama, rectángulos proporcionales al perímetro.

La estructura de proyección se produce cuando los nudos estructurales marcan situaciones de tensión que, al ser unidas por líneas, van conformando su estructura de proyección. En el caso del rectángulo, los cuatro vértices, los cuatro puntos medios de cada lado y el centro. Las líneas, por otro lado, pueden generar figuras semejantes a las de origen pudiendo, a su vez, ser divididas de igual manera.

Este tipo de orientación colabora en la organización de los elementos dentro de un campo, ya sea, para trabajarlo con las diversas particiones o como una estructura virtual en su interior para posicionar elementos compositivos o para la generación de formas únicas.

Las formas pueden, a su vez, interrelacionarse entre sí, vinculándose para producir diversas lecturas. Se destacan el distanciamiento o separación de las formas entre sí, el toque que se da cuando las formas se aproximan hasta tocarse (se da el toque en los rectángulos que conforman la trama del museo), la superposición o acercamiento de las formas hasta cubrirse parcialmente una con la otra, la unión o adición donde las formas quedan reunidas convirtiéndose en una forma nueva y mayor, la sustracción que se da cuando una forma le sustrae a otra la porción de superposición, la coincidencia que se observa cuando dos formas iguales se acercan hasta coincidir en su superposición y, finalmente, la partición o encuadre de una forma conservando los elementos necesarios que permitan referir al punto de partida.

Estos campos organizativos permiten generar estructuras formales dando como resultado una unidad limitada, finita o una composición infinita y continua.

Los componentes de la trama visual arquitectónica aplicada en las paredes del museo pueden entenderse como un motivo único en un campo limitado dando como resultado de diseño una trama visual que se extiende hacia los bordes de los volúmenes translúcidos.

\section{La trama tridimensional como espacio de proyección de color:}

El museo de la Fondation Louis Vuitton ha sido el soporte espacial para mostrar al mundo la obra de diferentes artistas visuales.

Uno de ellos, el artista conceptual Daniel Buren, provocó un juego de colores, proyecciones, transparencias y luces en toda la superficie tramada del Museo con su obra "Observatorio de la luz" convirtiéndolo en un ícono visual.

El concepto de esta obra se encuentra inspirado en la creación y en los encuentros innovadores que evoca la fundación Vuitton (Figura 6). 


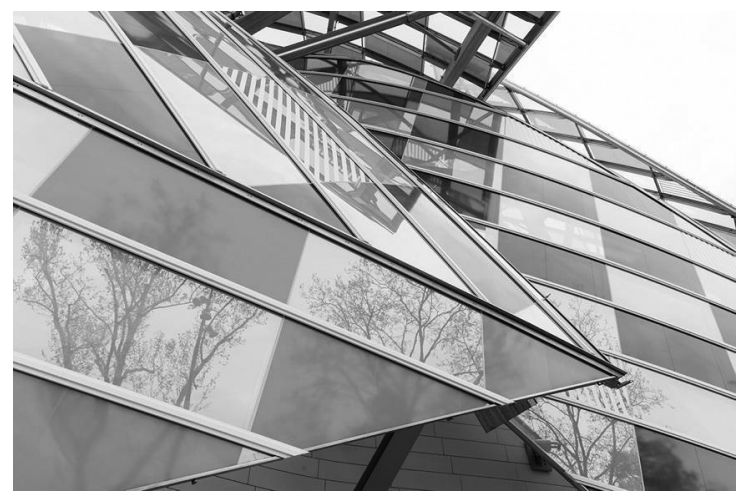

Figura 6. Trama con aplicación de color. Fuente: http:/ fondationlouisvuitton.fr/

El artista trabajó cubriendo con filtros de colores translúcidos los diversos módulos de trama que recubren las doce velas o volúmenes del edificio y que poseen 3600 piezas de vidrio creando, de este modo, un efecto caleidoscópico. Los colores seleccionados fueron trece y se aplicaron en forma de tablero de ajedrez en todas las fachadas otorgando un efecto óptico en constante cambio. Desde el exterior, estos paneles recogen tonalidades y texturas del entorno adaptándose a las horas del día y época del año creando una experiencia multidimensional.

La aplicación del color en algunos de los módulos de la trama envolvente permite potenciar el mensaje del museo creando un fuerte estímulo visual. El juego de contrastes entre los diversos cromas y las formas rectangulares logra formar una unidad que transmite sensaciones y emociones de gran riqueza visual.

\section{El Museo Foundation Luis Vuitton como lugar de experiencia emocional}

La llamada experiencia emocional es uno de los factores psicológicos de satisfacción del público. Se experimenta a través de diversas sensaciones y como experiencias vividas en la realidad en los lugares donde el público asiste aplicando estrategias de interacciones que hacen vivir a los visitantes experiencias emocionales distintas basadas en la idea de calidad generalizada.

Este museo, con sus características arquitectónicas innovadoras, tiene la capacidad de presentarse en un marco y espacio único. Es en sí mismo un lugar de identidad donde el público experimenta diversas sensaciones aún antes de ingresar. La visualización del edificio, con sus velas en movimiento permitiendo la reflexión del paisaje que lo rodea, agua, bosques, jardines, inician al visitante en una experiencia visual placentera. En el ingreso, el hall de entrada sirve para entrar al museo y al jardín simultáneamente convirtiéndose en un espacio multiuso a gran escala. Cuando el visitante camina en el interior del edificio, 
moviéndose de galería en galería, puede apreciar las hermosas vistas del jardín a través de los paneles vidriados y admirar las velas de cristal desde las escaleras exteriores.

En todos estos espacios intervienen los recursos estratégicos, creativos y tecnológicos que son capaces de generar múltiples sensaciones y experiencias estéticas y emocionales innovadoras en el público visitante.

Este museo se ha convertido en un lugar de experiencia directa y real con la empresa. Las experiencias, emociones, sensaciones y vivencias sólo se generan en el lugar donde se obtienen los servicios o los productos. Es allí donde se viven las emociones, en este entorno envolvente y transparente, con una arquitectura innovadora, es donde se establece una relación interactiva con la empresa misma, siendo indispensable para una fidelización duradera. Así lo plantea Joan Costa al analizar la fusión entre señalética e identidad:

En el marco natural, que es el paisaje urbano, espacio de acción y escenario de encuentros, la arquitectura corporativa es el envolvente y el contenido, al mismo tiempo, de la Identidad, la Cultura de la organización y la Calidad de sus bienes y servicios (Costa, 2007, p. 117).

El museo ha sido preparado estratégicamente como espacio, como lugar para que la experiencia emocional se produzca. Es el lugar de la identidad, lleno de magia y emoción donde el público se convierte en protagonista y no en un simple espectador.

\section{Nota}

1. En este trabajo se emplea el término hito para referirse a una señal clavada en el suelo que sirve para marcar el límite de un territorio o de una propiedad, o para indicar las distancias o la dirección en un camino.

2. En Diseño Gráfico, el módulo se corresponde a aquellas formas idénticas o similares entre sí que aparecen más de una vez en el diseño tendiendo a unificarlo.

3. Se emplea el término dintorno para hacer referencia a la delineación de las partes de una figura contenidas dentro de su contorno o de las contenidas en el interior de la planta o de la sección de un edificio.

\section{Bibliografía}

Arnheim, R. (2017). Arte y percepción visual. Alianza Editorial.

Chaves, N. (1990). La imagen corporativa. Barcelona: G. Gili.

Chaves, N. y Belluccia, R. (2003). La marca corporativa: gestión de símbolos y logotipos. Buenos Aires: Paidós.

Costa, J. (2005). Imagen corporativa en el siglo XXI. Buenos Aires: La Crujía.

Costa, J. (1989). Imagen Global: evolución del diseño de identidad. Barcelona: CEAC.

Costa, J. (2007). Señalética corporativa. Barcelona: Costa Punto Com Editor.

Costa, J. (2007). Señalética. Barcelona: CEAC. 
Frutiger, A. (1981). Signos, símbolos, marcas, señales. Barcelona: Gili.

Fondation Louis Vuitton. (2020). Disponible en: http://www.fondationlouisvuitton.fr/

González Ruiz, G. (1994). Estudio de diseño sobre la construcción de las ideas y su aplicación a la realidad. Buenos Aires: Emecé.

Muñoz, P. (2007). Simetría sutil, ¿Monstruos o formas? Cuadernos de la forma 8. Buenos Aires: CEMA.

\begin{abstract}
In the design of a High Complexity Visual Identity System, the use and application of a repertoire of secondary identifiers gives value to the identity itself.

The application of two-dimensional frames applied to three-dimensional spaces effectively impacts the identity of the company, redefining the Visual System itself, turning the architectural space into a corporate signage milestone within the Three-Dimensional Program. Currently, recognized clothing brands resort to mastering two-dimensional space with the aim of becoming universal graphic symbols with high power of remembrance within an urban space.
\end{abstract}

Keywords: archigraphy - two-dimensional - structure - geometry - landmark - identity module - identity signage - plot - three-dimensional.

Resumo: Na concepção de um Sistema de Identidade Visual de Alta Complexidade, a utilização e aplicação de um repertório de identificadores secundários valoriza a própria identidade.

A aplicação de molduras bidimensionais aplicadas a espaços tridimensionais impactam efetivamente a identidade da empresa, redefinindo o próprio Sistema Visual, tornando o espaço arquitetônico um marco da sinalização corporativa dentro do Programa Tridimensional.

Atualmente, marcas de vestuário reconhecidas recorrem ao domínio do espaço bidimensional com o objetivo de se tornarem símbolos gráficos universais com alto poder de lembrança dentro de um espaço urbano.

Palavras chave: arquigrafia - bidimensional - estrutura - geometria - marco - identidade - módulo - sinalização de identidade - trama - tridimensional.

[Las traducciones de los abstracts fueron supervisadas por el autor de cada artículo] 